\title{
Genomic Profiling in Patients With Malignant Peripheral Nerve Sheath Tumors Reveals Multiple Pathways With Targetable Mutations
}

\author{
Henry G. Kaplan, MDª Steven Rostad, MD ; Jeffrey S. Ross, MDc; Siraj M. Ali, MD; and Sherri Z. Millis, PhDc
}

\begin{abstract}
Background: The aim of this study was to determine the frequency of alterations in BRAF and other RAS/RAF genes, as well as other targetable pathways in malignant peripheral nerve sheath tumors (MPNSTs). Patients and Methods: Pathology specimens were available for 2 cohorts: (1) patients with MPNST at Swedish Cancer Institute $(n=17)$ from 2004 through 2016, and (2) patients with MPNST evaluated for >300 genomic alterations at Foundation Medicine from 2014 through 2016 ( $n=186$; including 2 Swedish patients with BRAF-mutated MPNST). Results: Of 201 MPNSTs, 13 (6.5\%) demonstrated BRAF alterations. In the Foundation Medicine cohort, 10 of 84 tumors (11.9\%) with no NF1 alterations had BRAF mutations ( 5 were V600E, 5 other), as did 3 of $102(2.9 \%)$ tumors with NF1 alterations (1 V600E, 2 other). In the Foundation Medicine cohort, $47 \%$ of patients had an alteration in at least one other gene in the RAS/RAF pathway (not including NF1 or BRAF); $46 \%$ had alterations in the PI3 pathway, with $70 \%$ having alterations in at least 1 of the 2 pathways; $57 \%$ had a CDKN2A alteration ( $80 \%$ in BRAF-mutated and $71 \%$ in NF1-altered patients); and $70 \%$ had an alteration in DNA repair genes. MPNST, both NF1 wildtype and NF1-mutated, often harbor alterations in the RAS/RAF pathway as well as changes related to DNA repair and CDKN2A/B. V600E and other mutations occur in $B R A F$, suggesting the need for second-generation activating $B R A F$ inhibitors. The concurrence of $B R A F$ and/ or NF1 alterations with CDKN2A/B mutations, in particular, may be significant in the transformation of neurologic tumors from benign to malignant. Conclusions: All MPNSTs would benefit from a comprehensive genomic analysis. Treatments targeted to RAS/RAF, DNA repair, and $C D K N 2 A / B$ pathways should be used and/or developed to treat this uncommon tumor.
\end{abstract}

J Natl Compr Canc Netw 2018;16(8):967-974 doi: 10.6004/jnccn.2018.7033

Malignant peripheral nerve sheath tumors (MPNSTs) are rare tumors for which no established systemic treatment exists. MPNSTs occur in approximately $10 \%$ of patients with neurofibromatosis type 1 , with malignancy usually present in the third or fourth decade of life. ${ }^{1}$ When MPNSTs occur sporadically in the absence of neurofibromatosis, they tend to present later in life, usually in the sixth or seventh decade, and often without an inactivating alteration in neurofibromin (NF1), which is seen in patients with neurofibromatosis type 1 . Wild-type NF1 protein exhibits an inhibitory

\footnotetext{
aSwedish Cancer Institute and bellNetix Corp, Seattle, Washington; and 'Foundation Medicine, Inc., Cambridge, Massachusetts.

Submitted December 23, 2017; accepted for publication April 11, 2018.

Drs. Ross, Ali, and Millis are employees of Foundation Medicine, Inc.

Drs. Ali and Ross have equity interest in Foundation Medicine, Inc. The remaining authors have disclosed that they have no financial interests, arrangements, affiliations, or commercial interests with the manufacturers of any products discussed in this article or their competitors.
}

effect on the RAS/RAF pathway. Such tumors exhibit increased activity in the RAS/RAF pathway by the lack of negative feedback. ${ }^{2}$ Alterations in the PI3K/PTEN/ AKT pathway can also affect RAS/RAF activity ${ }^{3}$ and can be stimulated by similar stimuli as the RAS/RAF pathway.

Previously, we reported on a patient with a sporadic MPNST, whose tumor featured a BRAF V600E mutation. ${ }^{4}$ This mutation results in activation of BRAF, leading to increased activity of the RAS/RAF pathway. ${ }^{5}$ This patient had a dramatic response to vemurafenib, an

This study was supported in part by the Kaplan Cancer Research Fund. Author contributions: Study concept and design: Kaplan, Ali, Millis. Development and methodology: Kaplan, Millis, Rostad. Data analysis and interpretation: Millis. Manuscript preparation: All authors. Critical revision: All authors.

Correspondence: Henry G. Kaplan, MD, Swedish Cancer Institute, 1221 Madison Street, Seattle, WA 98104.

Email: Hank.kaplan@swedish.org 
Kaplan et al

inhibitor of BRAF V600E. As a result, in the current study we analyzed the presence of BRAF alterations, other RAS/RAF alterations, and other genomic alterations in all MPNSTs studied at Swedish Cancer Institute and Foundation Medicine during the past 12 years.

\section{Patients and Methods}

Patients from the Swedish Cancer Institute pathology archives were identified under an Institutional Review Board (IRB)-approved protocol (No. SWD5531S-14). MPNSTs were studied using the QIAGEN QIAamp DNA FFPE Tissue Kit and BRAF mutation was analyzed using a laboratorydeveloped, allele-specific, real-time PCR assay to detect the major BRAF mutations; less common alterations, fusions, and large insertions/deletions are not identified by this assay. Patients from the Foundation Medicine archives underwent genome testing as previously reported. ${ }^{6,7}$ The NF1 gene was interrogated for all 4 classes of alterations: singlebase changes, copy number amplification, rearrangements, and large insertions/deletions (up to $\approx 25$ base changes) across all exons; sporadic versus neurofibromatosis-associated MPNST status was not evaluated. BRAF alterations were identified across all 4 classes of alterations and all exons. Approval for the Foundation Medicine cohort, including a waiver of informed consent and HIPAA waiver of authorization, was obtained from the Western IRB (Protocol No. 20152817). Clinical treatment and outcomes were not available for the Foundation cohort. However, pathology specimens from all BRAF-altered tumors were further reviewed and shown to be weakly S100-positive, melanin-negative, and HMB45-negative; none exhibited hypermutation. The 2 patients from the Swedish cohort with BRAF mutations received complete genomic analysis at Foundation Medicine and are included in the analysis of the Foundation cohort.

\section{Results}

A total of 201 patients with MPNSTs were included in this study, 17 of whom were identified at Swedish Cancer Institute; of these patients, 2 had BRAF alterations and were studied more extensively at Foundation Medicine.
Among the 186 MPNSTs profiled at Foundation Medicine (Table 1), 102 showed NF1 alterations (55\%) and 84 were NF1 wild-type (45\%). Of the patients with NF1 wild-type MPNST, 10 of 84 (12\%) exhibited BRAF alterations. Of the $10 \mathrm{BRAF}$ alterations, 9 were known to be pathogenic, including 5 BRAF V600E, 1 R389C, 1 I710fs*24, and 2 rearrangements (see supplemental eAppendix 1 for references, available with this article at JNCCN.org). One (P341I) was of unknown functionality. Among 102 patients with NF1 alterations, 2 exhibited likely pathogenic BRAF mutations, R389C and A762V, and one exhibited a functionally unknown G652E alteration.

Of the Foundation Medicine cohort, 46\% of patients had alterations in the PI3 pathway (only pathogenic mutations were counted), $57 \%$ had CDKN2A alterations, and $70 \%$ had an alteration in DNA repair genes. The cohorts of 102 NF1-mutated patients and 84 NF1 wild-type patients analyzed at Foundation Medicine were compared, with separate breakout of the 10 BRAF-altered cases. Differences in alteration rates were evident (Figures 1 and 2). Of note, CDKN2A was altered at a significantly higher rate in NF1-altered and BRAF-altered tumors versus non-NF1/BRAF $(71 \%, 80 \%$, and $34 \%$, respectively). Rates of TP53 (32\% of NF1, and only $14 \%$ of

\begin{tabular}{|c|c|c|}
\hline NF1 Status & BRAF Alterations & Functional Status $^{b}$ \\
\hline \multirow[t]{6}{*}{ Wild-type $(n=84)$} & V600E, $n=4$ & Pathogenic \\
\hline & $\begin{array}{l}\text { V600E and exon } \\
\text { duplication, } n=1\end{array}$ & Pathogenic \\
\hline & P341I, n=1 & Unknown significance \\
\hline & $\begin{array}{l}\text { Rearrangement, } \\
n=2\end{array}$ & Pathogenic \\
\hline & $1710 f s * 24, n=1$ & Pathogenic \\
\hline & $\mathrm{R} 389 \mathrm{C}, \mathrm{n}=1$ & Likely pathogenic \\
\hline \multirow{3}{*}{$\begin{array}{l}\text { NF1 alteration, } \\
\text { somatic or germline } \\
(n=102)\end{array}$} & $\mathrm{R} 389 \mathrm{C}, \mathrm{n}=1$ & Likely pathogenic \\
\hline & $\mathrm{A} 762 \mathrm{~V}, \mathrm{n}=1$ & Likely pathogenic \\
\hline & G652E, n=1 & Unknown significance \\
\hline
\end{tabular}

ancludes only patients whose tissue was submitted for comprehensive genomic profiling at Foundation Medicine ( 2 from the Swedish cohort and 184 others).

${ }^{b}$ Functional status identified through several databases, including COSMIC, 1000 Genomes Project, dbSNP, and ExAC (to filter out common germline variants). Published literature and conference proceedings were also used to assess functional significance of variants, adjusting default rules as needed to reflect whether a variant is likely pathogenic. 


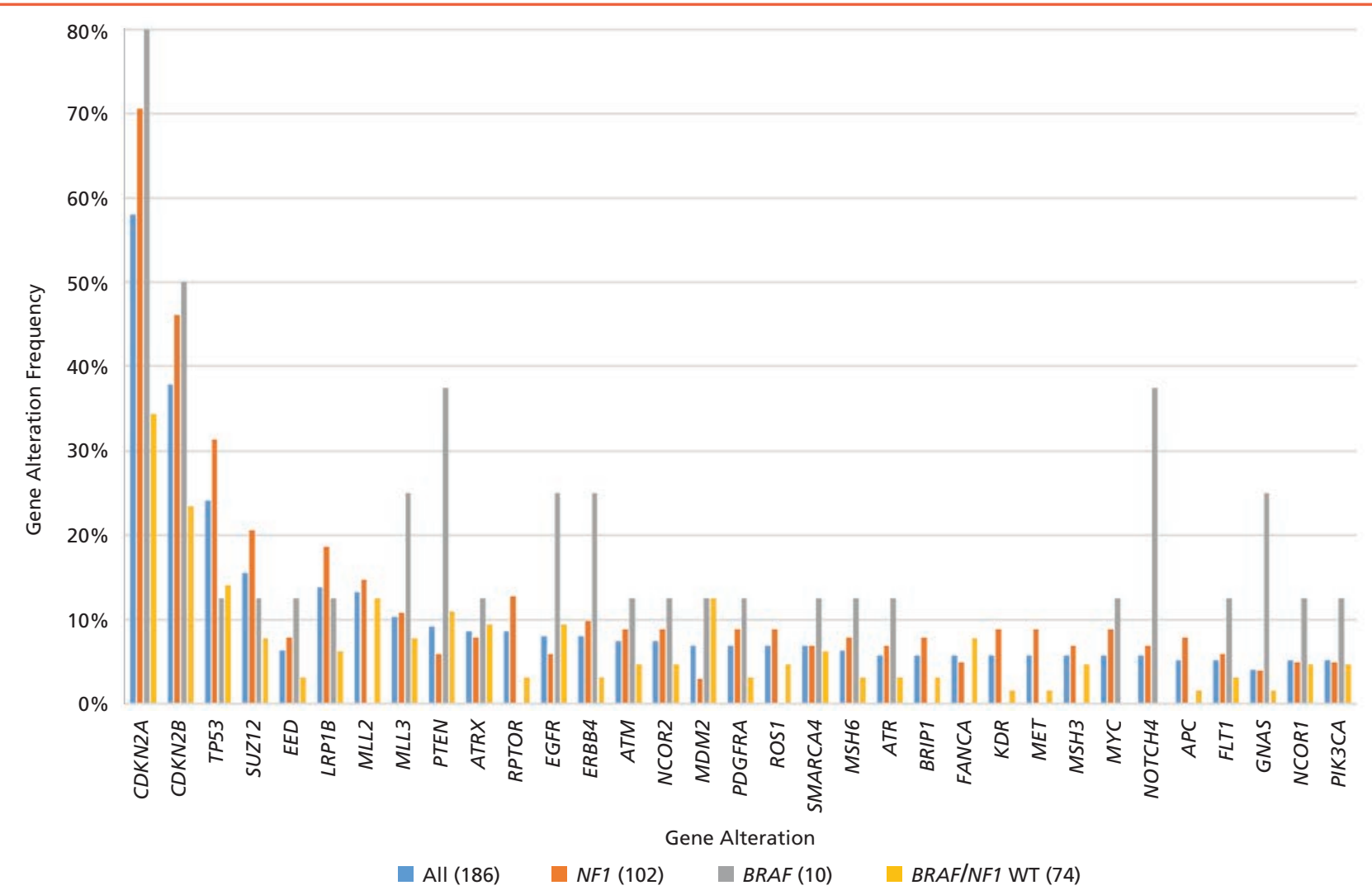

Figure 1. Comparison of frequency of top 50 genes altered in malignant peripheral nerve sheath tumor cases in the cohort analyzed at Foundation Medicine, with separate breakout of the BRAF and NF1-altered cases.

Abbreviation: WT, wild-type

non-NF1 and $13 \%$ of BRAF), SUZ12 (20\% of NF1altered, $13 \%$ of BRAF-altered, $9 \%$ of non-NF1/ non-BRAF-altered), and EED ( $8 \%$ of NF1-altered, $13 \%$ of BRAF-altered, $3 \%$ of non-NF $1 /$ non-BRAFaltered) also varied with subtype.

Across the entire Foundation Medicine MPNST cohort, $47 \%$ of tumors had an alteration in at least one other gene in the RAS/RAF pathway (ERBB2, ERBB3, ERBB4, KRAS, MET, HRAS, MAP2K1, MAP2K2, NRAS), not including NF1 or BRAF. By group, $52 \%$ of NF1-altered, $50 \%$ of BRAF-altered, and $38 \%$ of non-NF1/BRAF-altered had at least 1 RAS/RAF alteration (Figure 2). Most of the alterations in the RAS/RAF pathway are known alterations, for which targeted therapies have been associated with positive responses in other cancers. Among tumors with alterations in the PI3 pathway (AKT1, AKT2, FBXW7, INPP4B, MTOR, PIK3C2B, PIK3C2G, PIK3C3, PIK3CA, PIK3CB, PIK3CG, PIK3R1, PIK3R2, PTEN, RICTOR, STK11, TSC1, or TSC2), those with NF1 mutations had almost 2.5 times as many alterations in this pathway as those with non-NF1-altered; $70 \%$ of patients' tumors had alterations in at least 1 of the 2 pathways. Alterations in DNA repair genes (ATM, BARD1, BRCA1/2, FANCx, PBRM1, CHEK2, MSH2, MSH3, MSH6, NBN, PBRM1, POLE, RAD51, RAD51C) were found equally in $70 \%$ of tumors across subgroups.

Other altered genes identified that might inform targeted therapy included KDR, KIT, PDGFR, and FGFR1 (7\% each) and NTRK1 and FGFR2 (5\% each) (see supplemental eAppendix 2 for references).

The specific NF1 alterations in the Foundation Medicine cohort were further evaluated for frequency of specific alterations (Table 2). Notably, 2 cases harbored 4 NF1 alterations each; 14 tumors had $>1$ NF1 alteration.

\section{Case Reports of Patients From the Swedish Cohort}

Patient 1 was a 51-year-old woman who presented with an MPNST. Her initial presentation and treat- 


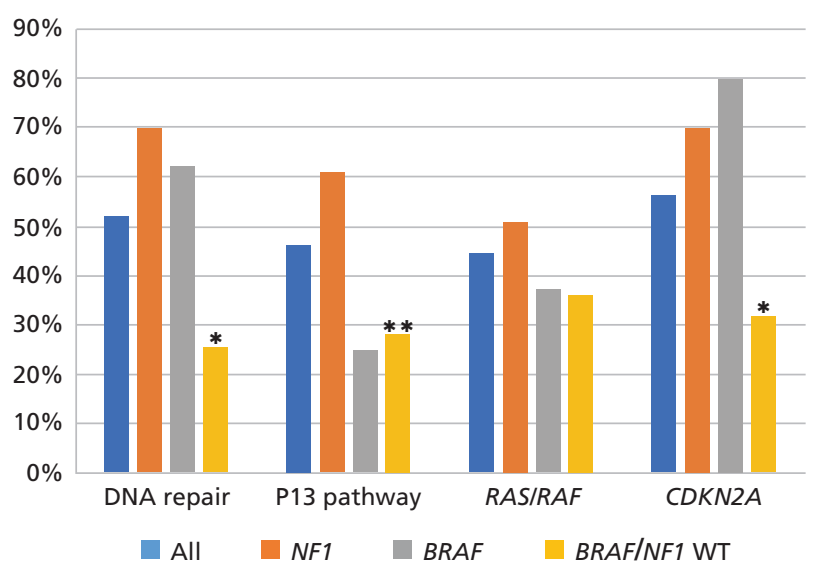

Figure 2. Ratio of patients in the Foundation Medicine cohort with malignant peripheral nerve sheath tumors exhibiting alterations in key pathways.

* Statistically significant difference between subtypes $(P<.0001)$. **Statistically significant differences between BRAF/NF1 wild-type (WT) and NF1-altered tumors $(P<.0001)$.

ment were previously reported, ${ }^{4}$ including a detailed pathologic description and clinical photographs documenting response. The present study provides details of further follow-up. She presented with a large, high right axillary mass. The tumor was intimately associated with the brachial plexus. She was treated with surgical removal and postoperative radiation therapy. Nine months later she developed a recurrence on the contralateral shoulder, which was also removed and radiated. This tumor was found to have a BRAF V600E

\begin{tabular}{|c|c|c|c|}
\hline Frequency & Protein Effect & $\begin{array}{l}\text { Known } \\
\text { Pathogenic? }\end{array}$ & Alteration \\
\hline 11 & Deletion & Known & $\mathrm{CN}$ \\
\hline 8 & Truncation & Likely & RE \\
\hline 4 & Rearrangement & Unknown & $\mathrm{RE}$ \\
\hline 3 & $q 514 f s^{2} * 43$ & Known & SV \\
\hline 3 & r1534* & Known & SV \\
\hline 3 & r1276* & Known & SV \\
\hline 2 & $\mathrm{q} 400 *$ & Known & sV \\
\hline 2 & r2258* & Likely & SV \\
\hline 2 & Splice site $7190-1 \mathrm{~g}>\mathrm{a}$ & Likely & sV \\
\hline 2 & y80* & Likely & sv \\
\hline 2 & q519* & Likely & SV \\
\hline 104 & $\begin{array}{l}\text { All different sites, } \\
\text { spanning length of } \\
\text { protein }\end{array}$ & $\begin{array}{l}15 \text { known, } \\
68 \text { likely, } \\
21 \text { unknown }\end{array}$ & $\begin{array}{l}103 \text { SV, } \\
\text { splice site, or } \\
\text { frame shift; } 1 \\
\text { amplification }\end{array}$ \\
\hline
\end{tabular}

Abbreviations: $\mathrm{CN}$, copy number change; RE, rearrangement; SV, single base variation. mutation. No clinical or genomic evidence of neurofibromatosis was seen. Shortly thereafter the patient developed widespread bulky metastatic disease. She had a transient modest response to sorafenib but a dramatic almost complete response to vemurafenib that lasted 4 months. At that time, she developed progressive disease. A second biopsy demonstrated a BRAF kinase domain (BRAF-KD) duplication. ${ }^{8}$ Therapy was discontinued for 1 month, followed by re-treatment with vemurafenib, this time with the addition of trametinib. She had a very good partial response, which lasted for 3 months. On disease progression, she was treated with vemurafenib plus everolimus without response. She experienced a 4-month partial response to carboplatin (her tumor was also BRCA2-mutated), but then developed progressive disease and died from her MPNST.

Patient 2 was a 58-year-old woman with a history of neurofibromatosis with multiple subcutaneous tumors. She developed a large tumor in the subcutaneous tissues of the occipital region. An 8-cm MPNST was surgically removed. Multiple nodules were present in the resection, which represented the benign plexiform neurofibroma characteristic of neurofibromatosis. Superimposed were varying degrees of malignant involvement by MPNST spreading within the confines of the perineurium of the plexiform neurofibroma. At its maximal involvement, the sarcoma was composed of a highly cellular population of monomorphous cells, which stained positively for S100. Sox 10 and p16 showed diffuse positive staining. BRAF V600E was detected in the malignant tumor. Analysis at Foundation Medicine revealed another BRAF alteration, which was identified at a very low frequency in a homopolymer repeat region of the gene, and could not be excluded as an artifact. Loss of CDKN2A/B was also detected. Focal areas of necrosis were seen. The Ki-67 labeling index was moderately elevated to $21 \%$, in contrast to nearly $0 \%$ in the benign neurofibromatosis regions. Tissue obtained from the neurofibroma at that time did not harbor either mutation. The sarcomatous component did not show extension to the soft tissue margins and was rather confined to the scaffolding of the plexiform neurofibroma. The peripheral nerve margins, however, showed, in addition to benign neurofibroma, rare pleomorphic and $\mathrm{Ki}-67$ positive nuclei, consistent with extension of the sarcoma to the nerve margins. Because of the positive surgical 
Genomic Profiling of MPNST

margin, the patient subsequently received 6,000 Gy of radiation therapy to the area. With 3 years' followup, no recurrence of this tumor has been seen. At 34 months after the appearance of the MPNST, the patient developed another large tumor mass, which on surgical removal demonstrated plexiform neurofibroma with focal atypia. She has received no further treatment for her disease at this time.

\section{Discussion}

MPNSTs are uncommon, occurring in approximately 1 in 10 million patients per year., ${ }^{9,10}$ They can sometimes be treated with local therapy, including surgery and radiation. However, these tumors can metastasize and be very aggressive, with an average long-term survival of only $20 \%$ to $50 \%$. The SARC006 trial reported that systemic chemotherapy with drugs used for sarcoma, such as doxorubicin, iphosphamide, and etoposide, produced approximately a $25 \%$ objective response rate, but these responses have not been durable. ${ }^{11}$ Trials with a variety of targeted agents are underway. Some responses have been reported with imatinib, an inhibitor of cKIT, but no clearly dramatic activity has been reported thus far. ${ }^{10}$ Therefore, new systemic treatments are sorely needed.

Approximately $50 \%$ of MPNSTs are considered to be derived from neurofibromas and possess an inactivating mutation in NF1, which would likely lead to increased RAS/RAF activity. Because alterations in the PI3K/PTEN/AKT pathway can also affect RAS/RAF activity, alterations in these pathways were also evaluated. In this series, PI3K alterations were significantly more common in NF1-altered versus NF1 wild-type tumors. During review, alterations in the DNA repair pathways were also identified frequently, suggesting that inability to correctly repair DNA damage caused an additive effect in malignancy. CDKN2A/B mutations were also extremely frequent, particularly in BRAF-mutated tumors.

Four other studies have reported activating BRAF V600E mutations in MPNST (Table 3). ${ }^{12-15}$ Combining these studies with our own (including the entire Swedish cohort), 20 of 351 patients (5.7\%) with MPNST demonstrated BRAF alterations, of which 12 of $351(3.4 \%)$ were the classic V600E mutation. In our study of the patients more fully analyzed at Foundation Medicine, $11.9 \%$ of NF1 wild-type MPNSTs harbored BRAF alterations, 5 of which were classic

\begin{tabular}{|c|c|c|c|}
\hline Reference & $\begin{array}{l}\text { Patients, } \\
\text { N }\end{array}$ & $\begin{array}{c}\text { All BRAF } \\
\text { Mutations, } \mathrm{n}\end{array}$ & $\begin{array}{c}\text { V600E } \\
\text { Mutations, }^{\text {a } n}\end{array}$ \\
\hline Schindler et $\mathrm{al}^{13}$ & 18 & 0 & 0 \\
\hline Botillo et al ${ }^{15}$ & 47 & 1 & 1 \\
\hline Je et $\mathrm{al}^{14}$ & 24 & 0 & 0 \\
\hline Hirbe et $a^{12}$ & 61 & 6 & 6 \\
\hline Kaplan & 201 & 13 & 5 \\
\hline Total & 351 & 20 & 12 \\
\hline
\end{tabular}

a $B R A F$ mutations other than V600E from the current series are noted in Table 1.

V600E activations (6.0\%), 4 were known or likely to be pathogenic, and 1 of unknown pathogenicity. Among NF1-mutated tumors, 2.9\% harbored a BRAF mutation, 2 of which were likely pathogenic and 1 was of unknown pathogenicity. This study, in agreement with that of Hirbe et al, ${ }^{12}$ suggests that BRAF alterations may be more common in patients with NF1 wild-type versus NF1-mutated tumors who develop MPNSTs. It is unclear whether methods used in the prior studies listed here would have detected BRAF mutations other than V600E. Our results demonstrate that, although uncommon, BRAF mutations are common enough that they should be searched for in all patients with MPNST.

In the one patient treated with a BRAF V600E inhibitor in this series, a remarkable response was seen. However, it lasted only approximately 4 months. After a month off treatment, she was rechallenged with the same drug plus an MEK inhibitor and briefly responded again. It is possible that the level of BRAF-mutated cells had decreased in her tumor to preresistant levels rendering the tumor again susceptible to vemurafenib, as has been reported and reviewed in mutated melanoma studies in both animals and humans. ${ }^{16-18}$ It is also possible that the addition of an MEK inhibitor was able to partially overcome tumor resistance by blocking the RAS/RAF pathway further downstream. ${ }^{19}$ The combination of a BRAF V600E blocker and an MEK inhibitor has been shown to be superior to single-agent therapy in melanoma. ${ }^{20,21}$ Subsequent treatment of this patient with vemurafenib and everolimus, an inhibitor of the mTOR/AKT pathway, was not effective, despite the demonstration of compensatory amplification of this alternate pathway as another mechanism of resistance to activated BRAF inhibitors. ${ }^{4}$ 
Kaplan et al

Patient 1 was also found to harbor a BRAF-KD duplication in addition to the V600E mutation when she was rebiopsied at the time of tumor progression. This mutation was sought in her original biopsy and was not present at that time. We have recently reported the occurrence of this mutation in a variety of tumor types, including patient $1 .^{8}$ It is possible that this mutation became as, or more, important to tumor survival than the V600E activation, and that this second mutation was partially overcome by the addition of an MEK inhibitor. Regorafenib has produced a dramatic response in one patient with this BRAF-KD mutation in a salivary acinic cell malignancy. ${ }^{8}$ The patient whose tumor harbored the fusion alteration treated with regorafenib in that study did not have the V600E mutation, suggesting that regorafenib may well be active against the kinase duplication mutation. Hutchinson et $\mathrm{al}^{22}$ also reported the development of 2 different BRAF fusion mutations in patients with melanoma. In that study, therapy with an MEK inhibitor in vitro was active in inhibiting tumor growth. Further, Kulkarni et al $^{23}$ described BRAF V600E-mutated melanoma in a patient who developed an AGAP3-BRAF fusion mutation that was resistant to vemurafenib but responded to vemurafenib plus an MEK inhibitor. After treatment was discontinued, the fusion mutation was no longer detectable, suggesting that such mutations may be clonally treatment-selected by exposure to BRAF inhibitors alone.

In our study, we identified a number of BRAF mutations in addition to BRAF V600E and the fusion mutation described earlier, many of which have been shown to be pathogenic (supplemental eAppendix 1). Chakraborty et $\mathrm{al}^{24}$ showed in Langerhans cell histiocytosis that an additional BRAF fusion mutation and other small in-frame BRAF deletions can lead to BRAF activation. These tumors are resistant to traditional BRAF V600E inhibitors but sensitive to MEK inhibitors, as well as the secondgeneration BRAF inhibitor PLX8394. Similarly, Diamond et $\mathrm{al}^{25}$ recently reported in histiocytosis that first-generation BRAF inhibitors are clinically effective in BRAF V600-mutant disease, whereas MEK inhibition was very successful against such patients harboring a variety of BRAF mutations, as well as altered MAP2K1, MAP2K2, KRAS, CRAF, and ARAF. Yao et $\mathrm{al}^{26}$ have studied the mechanism of BRAF activation in various BRAF mutants. Acti- vating BRAF V600 mutants are activated monomers when RAS activity is low and are inhibited by firstgeneration BRAF V600 blockers, whereas other activating $B R A F$ mutants noted in their study were constitutive RAS-independent dimers. First-generation BRAF blockers are effective against mutant monomers but not dimers. Their binding to one site in the dimer significantly reduces their affinity for the second, rendering those mutants resistant. Alternatively, the second-generation BRAF inhibitor PLX8394 binds to both sites of mutant BRAF dimers and is active against both monomer and dimer BRAF mutants. Although none of the non-V600 BRAF mutations reported in the current study were tested by Yao et al, it will be important to explore use of this drug in such patients. A clinical trial in patients exhibiting these types of mutations is currently underway (ClinicalTrials.gov identifier: NCT02428712).

Use of MEK inhibitors is particularly interesting in patients with loss of neurofibromin activity, which results in unrestrained activity of the RAS/RAF pathway. Multiple studies have suggested loss of neurofibromin activity as a driver of growth in melanomas that can be blunted by MEK inhibitors. ${ }^{27-30}$ The clinical utility of MEK inhibition was confirmed in the study by Dombi et al, ${ }^{31}$ in which $71 \%$ of children with neurofibromatosis type 1 had clinically significant durable shrinkage of benign inoperable plexiform neurofibromas with selumetinib, a known MEK inhibitor. Whether inhibition of MEK will yield clinically significant responses in patients with somatic NF1 mutations in MPNSTs remains to be explored. A number of compounds are in clinical development in an attempt to block this pathway further downstream at the level of ERK. Morris et $\mathrm{al}^{32}$ reported activity of one such compound even in tumors with BRAF V600E and MEK ${ }^{p 124 L}$ overexpression and acquired resistance to both BRAF and MEK inhibitors, whereas Sullivan et $\mathrm{al}^{33}$ reported clinical activity of BVD-523 in MAPK-mutant tumors, including in patients with BRAF V600E and other $B R A F$-activating mutations.

The high percentage of patients who harbored at least one alteration in the RAS/RAF pathway suggests that therapy options might exist for a large subgroup of patients with MPNST, including BRAF-altered tumors for which we have shown response. As previously discussed, a variety of resistance mechanisms have been demonstrated either in animals or 
in vitro, suggesting that activation of other metabolic pathways can bypass the RAS/RAF pathway. ${ }^{4,5}$ Combining blockade of multiple interacting pathways such as RAS/RAF and PI3K/PTEN/AKT may also prove useful in the clinical setting. The other studies noted in Table 3 performed varying degrees of extensiveness of genomic testing. In particular, we have found additional BRAF alterations other than V600E that are pathogenic. It will be important for future studies to perform genomic evaluation of all the relevant pathways discussed.

These considerations may prove useful in multiple types of tumors. As noted, activating BRAF mutations and BRAF fusions and alterations in other pathways that impact RAS/RAF activity have been reported in many malignancies. ${ }^{8,34}$ In addition, multiple kinases and transcription factors are known to stimulate this pathway both under normal and malignant conditions. Some of these, such as EGFR, have already been shown to be important in multiple tumor types, and inhibitors of these are clinically readily available. Such inhibitors could be evaluated both alone as well as in combination with the compounds discussed. ${ }^{35}$

Analysis of other pathways and driver genes identified alterations in DNA repair pathways, CDKN2A/B, the PI3 kinase pathway, ${ }^{36} \mathrm{KIT}, \mathrm{KDR}$, PDGFR, FGFR1/2/3, and NTRK1. The striking occurrence of $C D K N 2 A / B$ alterations in patients with BRAF and NF1 alterations may be of particular clinical significance. Studies have suggested that these 2 mutations taken together may be important in progression of pediatric low-grade glioma to secondary high-grade glioma, ${ }^{37-39}$ another neurologic malignancy developing from a less aggressive lesion, whereas Schiffman et $\mathrm{a}^{40}$ noted similar findings in pediatric malignant astrocytoma. Of note, patient 2 with BRAF-mutated MPNST also demonstrated loss of CDKN2A/B. She had a nearby benign neurofibroma that harbored neither mutation. The incidence of BRAF-activating mutations in benign neurofibromatosis tumors is not known. However, it is known that BRAF mutations are often seen in benign melanocytic nevi, ${ }^{41}$ wherein CDKN2A alteration has been shown to be a late step in the development of malignancy. ${ }^{42}$ Thus, it is likely that other events besides BRAF activation or NF1 deactivation, such as CDKN2A/B mutation, may be needed to confer malignant behavior. Indeed, Sohier et $\mathrm{al}^{43}$ hypothesized this very evolution in MPNST.

SUZ12 and EED mutations have been previously reported in MPNSTs. These are components of the polycomb repressive complex 2 (PRC2), and lossof-function mutations have been reported in high frequencies in these tumors, particularly in association with NF1 and CDKN2A alterations. ${ }^{44}$ Further, loss-of-function PRC2 mutations have been associated with amplification of RAS-driven transcription and vulnerability to MEK and BRD4 inhibitors. ${ }^{45}$ Our finding of an association between CDKN2A/B, BRAF, PRC2, and NF1 mutations is consistent with these studies, raising the question of a critical role in tumorigenesis. ${ }^{43}$ Trials are currently underway testing the utility of clinically available CDK4/6 inhibitors against this mutation. ${ }^{46}$

\section{Conclusions}

Comprehensive genomic evaluation of MPNSTs is warranted to identify not only BRAF alterations but also alterations in pathways related to RAS/RAF. The findings of frequent mutations in DNA repair genes and CDKN2A/B suggest additional targets for therapeutic intervention in this rare malignancy. ${ }^{47-50}$

\section{References}

1. Evans DG, Baser ME, McGaughran J, et al. Malignant peripheral nerve sheath tumors in neurofibromatosis. J Med Genet 2002;39:311-314.

2. Sun D, Tainsky MA, Haddad R. Oncogene mutation survey in MPNST cell lines enhances the dominant role of hyperactive Ras in NF 1 associated pro-survival and malignancy. Transl Oncologenomics 2012;5:1-7.

3. Yuan TL, Cantley LC. PI3K pathway alterations in cancer: variations on a theme. Oncogene 2008;27:5497-5510.

4. Kaplan HG. Vemurafenib treatment of BRAF V600E-mutated malignant peripheral nerve sheath tumor. J Natl Compr Canc Netw 2013;11:14661470.

5. Ascierto PA, Kirkwood JM, Grobb JJ, et al. The role of BRAF V600 mutation in melanoma. J Transl Med 2012;10:85-94.

6. Frampton GM, Fichtenholtz A, Otto GA, et al. Development and validation of a clinical cancer genomic profiling test based on massively parallel DNA sequencing. Nat Biotechnol 2013;31:1023-1031.

7. He J, Abdel-Wahab O, Nahas MK, et al. Integrated genomic DNA/ RNA profiling of hematologic malignancies in the clinical setting. Blood 2016;127:3004-3014.

8. Klempner SJ, Bordoni R, Gowen $\mathrm{K}$, et al. Identification of BRAF kinase domain duplications across multiple tumor types and response to RAFinhibitor therapy. JAMA Oncol 2016;2:272-274.

9. Ferner RE, Gutmann DH. International consensus statement on malignant peripheral nerve sheath tumors in neurofibromatosis. Cancer Res 2002;62:1573-1577.

10. Farid M, Demicco EG, Garcia R, et al. Malignant peripheral nerve sheath tumors. Oncologist 2014;19:193-201. 
11. Widemann BC, Reinke D, Helman L et al. SARC006: phase II trial of chemotherapy in sporadic and neurofibromatosis type 1 (NF1)-associated high-grade malignant peripheral nerve sheath tumors (MPNSTs) [abstract]. J Clin Oncol 2013;31(Suppl):Abstract 10522.

12. Hirbe AC, Pekmezci M, Dahiya S, et al. BRAFV600E mutation in sporadic and neurofibromatosis type 1-related malignant peripheral nerve sheath tumors. Neuro Oncol 2014;16:466-467.

13. Schindler G, Capper D, Meyer J, et al. Analysis of BRAF V600E mutation in 1,320 nervous system tumors reveals high mutation frequencies in pleomorphic xanthoastrocytoma, ganglioglioma, and extra-cerebellar pilocytic astrocytoma. Acta Neuropathol 2011;121:397-405.

14. Je EM, An CH, Yoo NJ, Lee SH. Mutational analysis of PIK3CA, JAK2, BRAF, FOXL2, IDH1, AKT1 and EZH2 oncogenes in sarcomas. APMIS 2012;120:635-639.

15. Bottillo I, Ahlquist T, Brekke H, et al. Germline and somatic NF1 mutations in sporadic and NF-1 associated malignant nerve sheath tumors. J Pathol 2009;217:693-701.

16. Mackiewicz-Wysocka M, Krokowicz L, Kocur J, Mackiewicz J. Resistance to vemurafenib can be reversible after treatment interruption. Medicine (Baltimore) 2014;93:e157.

17. McMahon M. Intermittent dosing in melanoma. Clin Adv Hematol Oncol 2015;13:348-350.

18. Schreuer M, Jansen $Y$, Planken $S$, et al. Combination of dabrafenib plus trametinib for BRAF and MEK inhibitor pretreated patients with advanced BRAF V600-mutant melanoma: an open-label, single-arm, duel-centre, phase 2 clinical trial. Lancet Oncol 2017;18:464-472.

19. Jessen WJ, Miller S, Jousma E, et al. MEK inhibition exhibits efficacy in human and mouse neurofibromatosis tumors. J Clin Invest 2013;123:340_ 347.

20. Chapman $P B$, Hauschild A, Robert $C$, et al. Improved survival with vemurafenib in melanoma with BRAF V600E mutation. N Engl J Med 2011;364:2507-2516.

21. Flaherty KT, Jeffrey $T$, Infante JR, et al. Combined BRAF and MEK inhibition in melanoma with BRAF V600 mutations. N Engl J Med 2012;367:1694-1703.

22. Hutchinson KE, Lipson D, Stephens PJ, et al. BRAF fusions define a distinct molecular subset of melanomas with potential sensitivity to MEK inhibition. Clin Cancer Res 2013;19:6696-6702.

23. Kulkarni A, Al-Hraishaw H, Simhadri S, et al. BRAF fusion as a novel mechanism of acquired resistance to vemurafenib in BRAFV600E mutant melanoma. Clin Cancer Res 2017;23:5631-5638.

24. Chakraborty R, Burke TM, Hampton OA, et al. Alternative genetic mechanisms of BRAF activation in Langerhans cell histiocytosis. Blood 2016;128:2533-2537.

25. Diamond EL, Durham BH, Dogan A, et al. Phase 2 trial of singe-agent cobimetinib for adults with BRAF V600-mutant and wild-type histiocytic disorders [abstract]. Blood 2017;130(Suppl 1):Abstract 257.

26. Yao Z, Torres NM, Tao A, et al. BRAF mutants evade ERK-dependent feedback by different mechanisms that determine their sensitivity to pharmacologic inhibition. Cancer Cell 2015;28:370-383.

27. Nissan MH, Pratilas,CA, Jones AM, et al. Loss of NF 1 in cutaneous melanoma is associated with RAS activation and MEK dependence. Cancer Res 2014;74:2340-2350.

28. Krauthammer M, Kong $Y$, Bacchiocchi A, et al. Exome sequencing identifies recurrent mutations in NF1 and RASopathy genes in sunexposed melanomas. Nat Genet 2013;47:996-1002.

29. Maertens $O$, Johnson B, Hollstein $P$, et al. Elucidating distinct roles for NF1 in melanogenesis. Cancer Discov 2013;13:338-349.
30. Whittaker SR, Theurillat JP, Van Allen E, et al. Genome-scale RNA interference screen implicates NF1 loss in resistance to RAF inhibition. Cancer Discov 2013;13:350-362.

31. Dombi E, Baldwin A, Marcus LJ, et al. Activity of selumetinib in neurofibromatosis type1-related plexiform neurofibromas. N Engl J Med 2016;375:2550-2560

32. Morris EJ, Jha S, Restaino CR, et al. Discovery of a novel ERK inhibitor with activity in models of acquired resistance of BRAF and MEK inhibitors. Cancer Discov 2013;3:742-750.

33. Sullivan RJ, Infante JP, Janku F, et al. First-in-class ERK 1-2 inhibitor ulixertinib (BVD-523) in patients with MAPK mutant advanced solid tumors: results of a phase I dose-escalation and expansion study. Cancer Discov 2018;8:184-195

34. Hyman DM, Puzanov I, Subbiah V, et al. Vemurafenib in multiple nonmelanoma cancers with BRAF V600 mutations. N Engl J Med 2015;373:726-736.

35. Yao YM, Donoho GP, Iversen PW, et al. Mouse PDX trial suggests synergy of concurrent inhibition of RAF and EGFR in colorectal cancer with BRAF or KRAS mutations. Clin Cancer Res 2017;23:5547-5560.

36. Mayer IA, Artega CL. The PI3K/AKT pathway as a target for cancer treatment. Annu Rev Med 2016;67:11-28.

37. Mistry M, Zhukova N, Merico D, et al. BRAF mutation and CDKN2A/B deletion define a clinically distinct subgroup of childhood secondary high grade glioma. J Clin Oncol 2015;33:1015-1022.

38. Lassaletta A, Zapotocky M, Mistry M, et al. Therapeutic and prognostic implications of BRAF V600E in pediatric low-grade gliomas. J Clin Oncol 2017;35:2934-2941.

39. Broniscer A. Malignant transformation of low-grade gliomas in children: lessons learned from rare medical events. J Clin Oncol 2015;33:978-979.

40. Schiffman JD, Hodgson JG, VandenBerg SR, et al. Oncogenic BRAF mutation with CDKN2A inactivation is characteristic of a subset of pediatric malignant astrocytomas. Cancer Res 2010;70:512-519.

41. Kumar R, Angelini S, Snellman E, et al. BRAF mutations are common somatic events in melanocytic nevi. J Invest Dermatol 2004;122:342-348.

42. Shain AH, Yeh I, Kovalyshyn I, et al. The genetic evolution of melanoma from precursor lesions. N Engl J Med 2015;373:1626-1636.

43. Sohier P, Luscan A, Lloyd A, et al. Confirmation of mutation landscape of NF1 associated malignant peripheral nerve sheath tumor. Genes Chromosomes Cancer 2017;56:421-426.

44. Lee $W$, Teckie $S$, Wiesner $T$, et al. PRC2 is recurrently inactivated through EED or SUZ12 loss in malignant peripheral nerve sheath tumors. Nat Genet 2014;46:1227-1232.

45. DeRaedt T, Beert E, Psamant E, et al. PRC2 subunits are tumor suppressors in NF1 deficient solid tumors. Nature 2014;514:247-251.

46. Sherr CJ, Beach D, Shapiro GI. Targeting CDK4 and CDK6: from discovery to therapy. Cancer Discov 2015;6:353-357.

47. Graziani $G$, Szabo C. Clinical perspectives of PARP inhibitors. Pharmacol Res 2005;52:109-118.

48. Isakoff SJ, Mahyer EL, Traina TA, et al. TBCRC009: a multicenter phase II clinical trial of platinum monotherapy with biomarker assessment in metastatic triple negative breast cancer. J Clin Oncol 2015;33:1902-1909.

49. Massacesi C, DiTomaso E, Urba P, et al. PI $3 \mathrm{~K}$ inhibitors as new cancer therapeutics: implications for clinical trial design. Onco Targets Ther 2016;9:203-210.

50. Levitski A. Tyrosine kinase inhibitors: views of selectivity, sensitivity, and clinical performance. Annu Rev Pharmacol Toxicol 2013;53:161-185. 